\title{
Nuclear masses near the proton drip-line and their impact on nucleosynthesis in explosive stars
}

\author{
SUN Yang \\ Department of Physics, Shanghai Jiao Tong University, Shanghai 200240, China
}

The interplay of the fundamental forces (here mainly the strong force and electromagnetic force) holds nucleons together to form atomic nuclei. However, in nature only less than ten percent of the total number of nuclei is stable. The majority of nuclei are unstable; that is, after having lived for a certain time, they change into the daughter nuclei by radioactive decay. The familiar decay types that can be found in most parts of the chart of nuclide are $\alpha$-decay and $\beta$-decay. There are other important processes in which unstable nuclei can change into other ones. These occur, for example, in very neutronrich or proton-rich regions of the chart of nuclide. There are boundaries called drip-lines, which are the lines on the $\mathrm{Z}$ (proton)-N (neutron) plane where the nucleon separation energy is zero. The nuclear binding becomes weaker and weaker when moving from the stable region towards the drip-lines, and eventually no nucleus can exist beyond the drip-lines. Taking the proton-rich region as an example, near the proton drip-line, protons are only weakly bound and a proton radioactivity can occur. Also because of the small binding, nuclei near the proton drip-line may capture additional protons and become new, heavier nuclides if the capture conditions are present. Detailed knowledge on the nucleon-capture processes lies at the heart of the understanding of nucleosynthesis in the Universe - a very fundamental but unanswered question about how the heavy elements (i.e. those heavier than iron) are created.

In order for nuclei to capture protons, a high temperature environment (usually above $1 \times 10^{9} \mathrm{~K}$ ) is needed so that they can overcome the large coulomb barrier for charged particle reactions. A hydrogen rich environment is also required to have a large proton flux ${ }^{[1]}$. The type-I $\mathrm{X}$-ray bursts, which are explosive consequences of accreting neutron stars ${ }^{[2]}$, have been suggested to be the physical sites where the nucleosynthesis rp-process ${ }^{[3]}$ (the rapid proton-capture process) takes place. The rpprocess consists of consecutive proton captures onto seed nuclei plus some $\beta^{+}$-decays to produce new elements, which proceed in the proton-rich nuclei near the proton drip-line. Understanding the nucleosynthesis clearly requires a detailed analysis of the reaction path and the reaction flow in the whole mass range involved in the process. Particularly important for rp-process nuclear reaction network calculations are the nuclear masses of the proton-rich isotopes because these determine the $Q$ values for proton-capture and $\beta^{+}$-decay. $\mathrm{Nu}^{-}$ clear mass is such a sensitive and dominant quantity in the discussion that the quality and predictive power of the astrophysical models for nucleosynthesis depend crucially on the accuracy of the mass. However, only a very limited amount of experimental mass is presently available for proton-rich unstable nuclei with $A>60$ because of extreme difficulty in performing experiments in laboratory for such short-lived isotopes. Theoretical masses based on various mass model predictions are not reliable, partly due to the rather complex nuclear structure in the proton-rich nuclei, which itself is a current frontier problem in nuclear physics.

Fortunately, not all the isotopes lying on the reaction path are equally important. It has been suggested ${ }^{[3]}$ that there are some key nuclei on the rp-process path that most strongly influence the nucleosynthesis products.

doi: 10.1007/s11434-009-0704-3

email: sunyang@sjtu.edu.cn 
Accurate masses of the key nuclei are therefore the primarily desired quantities. The mass of ${ }^{65} \mathrm{As}$, for example, has been frequently mentioned from the early years ${ }^{[1]}$ till today ${ }^{[4]}$, because the uncertainty in the $Q$-values of the proton-capture reactions ${ }^{64} \mathrm{Ge}(\mathrm{p}, \gamma){ }^{65} \mathrm{As}$ and ${ }^{65} \mathrm{As}(\mathrm{p}, \gamma)$ ${ }^{66} \mathrm{Se}$ have by far the largest effect in the astrophysical XRB model. In all the relevant discussions so far in the literature, the calculated value ${ }^{[5]}$ has been used for the ${ }^{65} \mathrm{As}$ mass, and all the conclusions have been based on the theoretical mass. With the recent commissioning of the Cooler Storage Ring at the Heavy Ion Research Facility in Lanzhou (HIRFL-CSR), direct mass measurement for the short-lived isotopes close to the proton drip-line becomes possible. In this issue, $\mathrm{Xu}$ and his colleagues report ${ }^{[6]}$ their measured masses for three short-lived nuclei $\left({ }^{63} \mathrm{Ge},{ }^{65} \mathrm{As}\right.$ and $\left.{ }^{67} \mathrm{Se}\right)$ from the first running experiment at HIRFL-CSR. These masses have

1 Champagne A E, Wiescher M. Explosive hydrogen burning. Ann Rev Nucl Sci, 1992, 42: 39-76

2 Bildsten $\mathrm{L}$. Thermonuclear burning on rapidly accreting neutron stars. In: Buccheri R, van Paradijs J, Alpar M A, eds. The Many Faces of Neutron Stars. Dordrecht: Kluwer, 1998. 419

3 Schatz H, Aprahamian A, Goerres J, et al. rp-process nucleosynthesis at extreme temperature and density conditions. Phys Rep, 1998, 294: $167-264$

4 Parikh A, Jose $\mathrm{J}$, Iliadis C, et al. Impact of uncertainties in reaction $Q$ never been successfully measured by any laboratories. $\mathrm{Xu}$ et al. now demonstrate that the facilities newly constructed in Lanzhou, China, are able to perform this kind of experiment, which could provide excellent oppor tunities for China to lead several research directions in nuclear physics and nuclear astrophysics. The preliminary results for the measured masses presented in ref. [6] are interesting in that they would result in different conclusions as the prevalent believe. Unfortunately, the error bars prevent them from drawing an immediate conclusion. If the experimental uncertainties could be further reduced to a smaller amount, say within \pm 150 $\mathrm{keV}$, the result would be extremely significant. As pointed out by $\mathrm{Xu}$ et al.$^{[6]}$, this would change the current picture of the location of the proton drip-line, and strongly modify the so-far discussed results for the rp-process nucleosynthesis.

values on nucleosynthesis in type I X-ray bursts. Phys Rev C, 2009, 79: 045802

5 Audi G, Wapstra A H, Thibault C. The AME 2003 atomic mass evaluation: (II) Tables, graphs and references. Nucl Phys A, 2003, 729: $337-676$

6 Xu H S, Tu X L, Yuan Y J, et al. First mass measurement of shortlived nuclides at HIRFL-CSR. Chinese Sci Bull, 2009, 54: 47494752 